\title{
UNIFORM-FIELD ELECTRODES WITH MINIMUM WIDTH
}

\author{
Gerard J. ERNST \\ Department of Applied Physics, Twente University of Technology, Enschede, The Netherlands
}

Received 1 September 1983

\begin{abstract}
Simple analytic expressions are derived to design uniform-field electrodes that are superior in compactness and field uniformity to those described in literature. When such electrodes are used in a TEA laser system, smaller electrodes can be used for the same gas-discharge width.
\end{abstract}

In order to obtain high output powers from transversely excited pulsed lasers it is important to have a very uniform energy loading of the active gas medium. That is why there is a need for specially contoured electrodes that produce a very uniform field-strength distribution over a certain amount of surface. A number of authors tried to solve this problem. Rogowski's [1] and Chang's [2] profiles are most commonly used up to now. For some applications, however, a smaller electrode width is required for a given discharge width than will be obtained by e.g. Chang's formulas. This, for instance, is the case for large-aperture $\mathrm{CO}_{2}$ and $\mathrm{CO}$ lasers, where the distance of the $\mathrm{UV}$ source from the electrode centre is an important measure, or for large-aperture UV lasers, where the electrode inductance contributes significantly to the total circuit inductance. Recently [3] Chang profiles have been improved by modification of the conformal transformation. Although those profiles are excellent as for their specifications, the analytic expressions are rather complicated, which limits their use.

We present here a family of analytic profiles for uniform-field electrodes that have minimum width and can produce almost any degree of field-strength uniformity at the electrode surface, and have analytic expressions as simple as Chang's. We start with the following conformal transformation:

$\zeta=w+k_{0} \sinh w+k_{1} \sinh 2 w$,

where $\xi=x+\mathrm{i} y$ and $w=u+\mathrm{i} v$, with $x$ and $y$ being the space coordinates and $u$ and $v$ being the flux and potential functions, respectively. For each value of $v(|v|<\pi)$, the profile of the corresponding equipotential surface is given by

$x=u+k_{0} \sinh u \cos v+k_{1} \sinh 2 u \cos 2 v$,

$y=v+k_{0} \cosh u \sin v+k_{1} \cosh 2 u \sin 2 v$,

where $u$ is the running variable. This profile is symmetric with respect to the $y$ axis and the $+v$ and $-v$ equipotentials are mirror images with respect to the $x$ axis, which are prerequisites for a uniform-field electrode. From relations (2) and (3) it can be seen that the profiles are not uniquely determined. Three independent variables, $k_{0}, k_{1}$, and $v$, determine the form of the profile as well as the electric-field strength distribution. Once $k_{0}$ has been chosen as an independent variable, $k_{1}$ and $v$ can be used to optimize the electric-field strength distribution over the surface. To find the optimum profile an expression is needed for the electric-field strength:

$$
\begin{aligned}
E^{-2} & =|\mathrm{d} \zeta / \mathrm{d} w|^{2}=\left|1+k_{0} \cosh w+2 k_{1} \cosh 2 w\right|^{2} \\
& =f^{2}(u)+g^{2}(u),
\end{aligned}
$$

with

$f(u)=1+k_{0} \cosh u \cos v+2 k_{1} \cosh 2 u \cos 2 v$,

$g(u)=\quad k_{0} \sinh u \sin v+2 k_{1} \sinh 2 u \sin 2 v$.

When the electric-field strength is expressed as a power-series expansion around $u=0$, 


$$
\begin{aligned}
E & =E_{0}\left(k_{0}, k_{1}, v\right)+E_{2}\left(k_{0}, k_{1}, v\right) u^{2} \\
& +E_{4}\left(k_{0}, k_{1}, v\right) u^{4}+\ldots,
\end{aligned}
$$

the optimum profile can be found by requiring the lower coefficients $E_{2}$ and $E_{4}$ to vanish or

$$
\begin{aligned}
E_{2} & =-\left[f(0) f^{(2)}(0)+\left(g^{(1)}(0)\right)^{2}\right] / f^{3}(0)=0 . \\
E_{4} & =-\left[f(0) f^{(4)}(0)+3\left(f^{(2)}(0)\right)^{2}\right. \\
& \left.+4 g^{(1)}(0) g^{(3)}(0)\right] / f^{3}(0)=0,
\end{aligned}
$$

where the exponent between brackets denotes the number of differentiations with respect to $u$.

The results of our computer calculations are shown in table 1 . As can be seen, the optimized values of $v$ deviate only very slightly from $\pi / 2$ up to $k_{0}$ values of 0.1 . For all practical profiles the $v$ value can therefore be approximated by $\pi / 2$. The value of $k_{1}$ can then be found from eq. (8) with $v=\pi / 2$. This results in

$k_{0}^{2}-8 k_{1}\left(1-2 k_{1}\right)=0$

or

$k_{1}=\frac{1}{4}-\frac{1}{4}\left(1-k_{0}^{2}\right)^{1 / 2}$,

which often can be approximated by

$k_{1}=\frac{1}{8} k_{0}^{2}$.

Table 1

Optimized values of $k_{0}, k_{1}$, and $v$ for the profile of eqs. (2) and (3)

\begin{tabular}{lll}
\hline$k_{0}$ & $k_{2}$ & $v$ \\
\hline 0.001 & $0.1250002 \mathrm{E}-6$ & 1.570796 \\
0.0015 & $0.2812508 \mathrm{E}-6$ & 1.570796 \\
0.002 & $0.5000025 \mathrm{E}-6$ & 1.570796 \\
0.003 & $0.1125013 \mathrm{E}-5$ & 1.570796 \\
0.005 & $0.3125098 \mathrm{E}-5$ & 1.570796 \\
0.007 & $0.6125375 \mathrm{E}-5$ & 1.570796 \\
0.01 & $0.1250156 \mathrm{E}-4$ & 1.570795 \\
0.015 & $0.2813292 \mathrm{E}-4$ & 1.570793 \\
0.02 & $0.5002505 \mathrm{E}-4$ & 1.570788 \\
0.03 & $0.1126271 \mathrm{E}-3$ & 1.570769 \\
0.05 & $0.3134887 \mathrm{E}-3$ & 1.570670 \\
0.07 & $0.6163444 \mathrm{E}-3$ & 1.570445 \\
0.1 & $0.1266441 \mathrm{E}-2$ & 1.569746 \\
0.15 & $0.2901734 \mathrm{E}-2$ & 1.567006 \\
0.2 & $0.5315494 \mathrm{E}-2$ & 1.560798 \\
0.3 & $0.1400316 \mathrm{E}-1$ & 1.514851 \\
\hline
\end{tabular}

So, the resulting profile is given by

$x=u-\frac{1}{4}\left[1-\left(1-k_{0}^{2}\right)^{1 / 2}\right] \sinh 2 u$,

$y=\pi / 2+k_{0} \cosh u$,

where $u$ is the running variable and $k_{0}$ an independent variable that determines the ratio between the electrode gap and the width of the electrodes (or the gas discharge).

In fig. 1 the electrode profile is shown for a $k_{0}$ value of 0.02 according to eqs. (13) and (14) (curve B) and compared to Chang's profile (curve $A$ ). The exact profile found from eqs. (2) and (3) with optimized values for $k_{0}, k_{1}$ and $v$ as well as the profile from [3] with an eighth-power function for $k$ differ only slightly from curve B. All $x$ and $y$ values in the figures have been normalized to $y_{0}=\pi / 2+k_{0}$.

It can be seen that our profile is 10 to $15 \%$ smaller than Chang's ones, and the expressions are simpel and have only one free parameter. Moreover, the uniformity of the field-strength distribution over the electrode surface is greatly improved. This is demonstrated in fig. 2, where a plot has been made of the deviation of the field-strength at the electrode surface from its central value, normalized to this central value. Curve A relates to Chang's profile and the curves B, B', and $B^{\prime \prime}$ relate respectively to our new profile according to eqs. (13) and (14), the profile according to eqs. (2) and (3) and the profile from [3]. The curves B, $\mathbf{B}^{\prime}$,

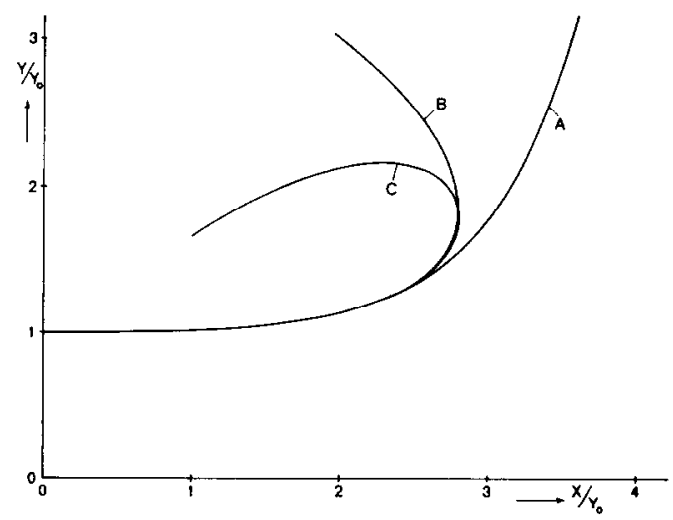

Fig. 1. The shape of the optimized profiles for three different cases. Curve A is Chang's profile, curve B relates to eqs. (13) and (14); curve $C$ to eqs. (16) and (17). For all cases $k_{0}=$ 0.02 . 


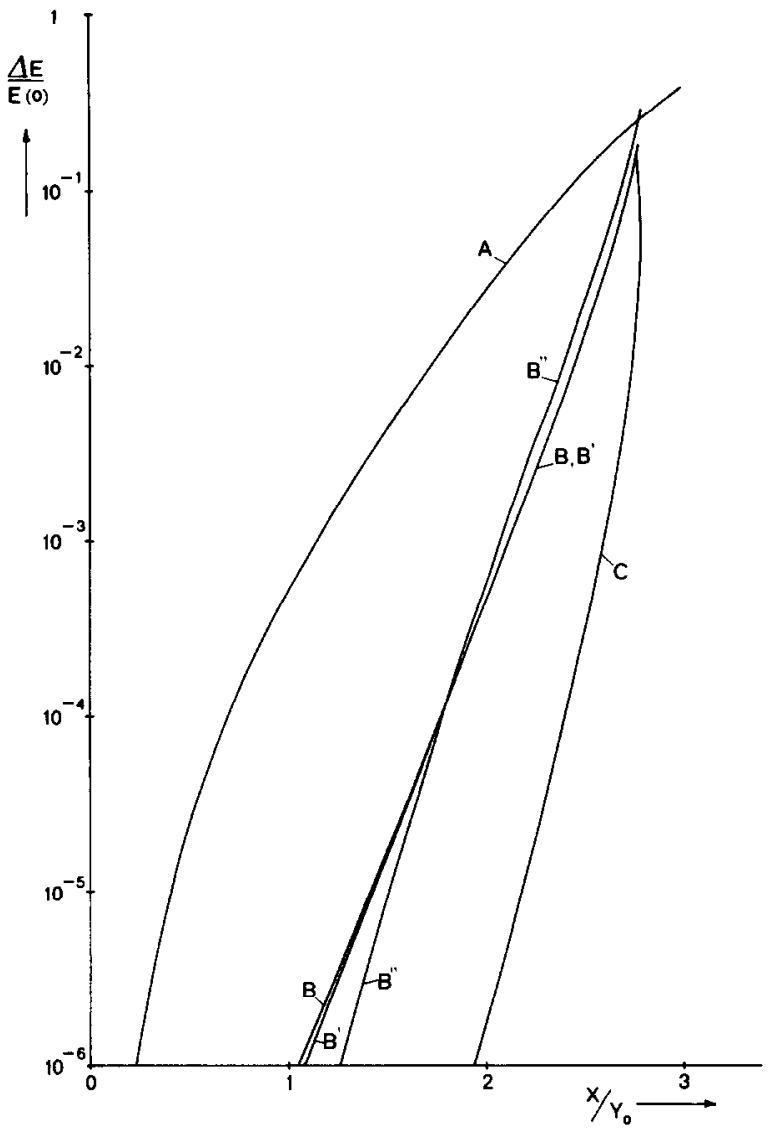

Fig. 2. The normalized deviation of the electric-field strength at the surface of the electrodes from the central value for Chang's profile (curve A), for the profile from eqs. (13) and (14) (curve B), for the profile from eqs. (2) and (3) (curve $B^{\prime}$ ), for the profile from [3] (curve B"), and for the profile from (16) and (17) (curve $C$ ), plotted against the normalized position at the electrode. For all cases $k_{0}=0.02$.

and $\mathrm{B} "$ all lie close together and show a much better field-strength uniformity than curve A. So, it can be expected that our smaller profiles produce a wider gas discharge.

If necessary, further improvement of the profile is possible by adding extra terms in the conformal transformation, for instance:

$\zeta=w+k_{0} \sinh w+k_{1} \sinh 2 w+k_{2} \sinh 3 w$.

Now the parameters $k_{0}, k_{1}, k_{2}$, and $v$ can be used to further optimize the field-strength distribution by requiring all three coefficients $E_{2}, E_{4}$, and $E_{6}$ from eq.
(8) to vanish. The calculations show that the optimized value of $v$ lies closer to $\pi / 2$ than in the former case. For instance, for $k_{0}=0.02$ the optimized values for $k_{1}, k_{2}$, and $v$ are: $k_{1}=0.5000125 \mathrm{E}-4, k_{2}=$ $0.8334357 \mathrm{E}-7$, and $v=1.570796$. So, the profile is given by:

$x=u-k_{1} \sinh 2 u$

$y=\pi / 2+k_{0} \cosh u-k_{2} \cosh 3 u$,

and the optimized coefficients $k_{1}$ and $k_{2}$ can be found by requiring the coefficients $E_{2}$ and $E_{4}$ to vanish, which gives the cubic equations with the following roots:

$k_{1}=\frac{1}{4}\left\{1-\left[1-\left(k_{0}-9 k_{2}\right)^{2}\right]^{1 / 2}\right\}$,

$k_{2}=\frac{5}{81} k_{0}\left\{1-\left[1-\frac{9}{25}\left(1-8 k_{1} / k_{0}^{2}+64 k_{1}^{2} / k_{0}^{2}\right)\right]^{1 / 2}\right\}$.

The values of $k_{1}$ and $k_{2}$ can be easily found after some iteration steps. A good approximation often is

$k_{1}=\frac{1}{8} k_{0}^{2}$

$k_{2}=\frac{1}{90} k_{0}^{3}$.

A plot of this profile, with $k_{0}=0.02$, is shown in fig. 1 , curve $\mathrm{C}$, and its field-strength distribution is shown in fig. 2, curve C. Again, improvement of the uniformity of the field-strength distribution is found. Of course, further improvement can be achieved by adding extra higher-order terms in the conformal transformation, so that in principle any degree of uniformity in field strength at the electrode surface can be obtained. However, this will be of little practical importance. Moreover, it is obvious that our procedure, resulting in simple analytic formulas, give the minimum possible electrode width.

It should be pointed out that truncation of the electrodes according to eqs. (16) and (17), which can be done at the electrode backside, has only little influence on the uniformity of the field-strength distribution at the central part of the electrodes.

\section{References}

[1] W. Rogowski, Arch. Electrotech. 12 (1923) 1.

[2] T.Y. Chang, Rev. Scient. Instr. 44 (1973) 405.

[3] G.J. Ernst, Optics Comm. 47 (1983) 47. 